\title{
Phase I-II Study of Short-course Accelerated Radiotherapy (SHARON) for Palliation in Head and Neck Cancer
}

\author{
ELEONORA FARINA ${ }^{1}$, JENNY CAPUCCINI ${ }^{1}$, GABRIELLA MACCHIA ${ }^{2}$, LUCIANA CARAVATTA ${ }^{3}$, \\ NAM P. NGUYEN ${ }^{4}$, SILVIA CAMMELLI ${ }^{1}$, ANDREA FARIOLI ${ }^{5}$, GIUSEPPE ZANIRATO RAMBALDI ${ }^{6}$, \\ SAVINO CILLA ${ }^{7}$, TIGENEH WONDEMAGEGNHU ${ }^{8}$, A.F.M. KAMAL UDDIN ${ }^{9}$, MOSTAFÀ AZIZ SUMON $^{10}$, \\ DOMENICO GENOVESI ${ }^{3}$, MILLY BUWENGE ${ }^{1}$, FRANCESCO CELLINI $^{11}$, \\ VINCENZO VALENTINI ${ }^{11}$, FRANCESCO DEODATO $^{2 *}$ and ALESSIO G. MORGANTI ${ }^{{ }^{*}}$ \\ ${ }^{1}$ Radiation Oncology Center and ${ }^{6}$ Radiology Unit, Department of Experimental, \\ Diagnostic and Specialty Medicine (DIMES), S. Orsola-Malpighi Hospital, University of Bologna, Bologna, Italy; \\ ${ }^{2}$ Radiotherapy Unit, Department of Oncology, ${ }^{7}$ Medical Physics Unit, \\ Giovanni Paolo II Foundation, Catholic University of Sacred Heart, Campobasso, Italy; \\ ${ }^{3}$ Department of Radiation Oncology, SS. Annunziata Hospital, G. D'Annunzio University of Chieti, Chieti, Italy; \\ ${ }^{4}$ Department of Radiation Oncology, Howard University College of Medicine, Washington, DC, U.S.A.; \\ ${ }^{5}$ Department of Medical and Surgical Sciences (DIMEC), University of Bologna, Bologna, Italy; \\ ${ }^{8}$ Department of Radiotherapy, Black Lion Hospital, Addis-Ababa, Ethiopia; \\ ${ }^{9}$ Department of Radiation Oncology, United Hospital Limited, Dhaka, Bangladesh; \\ ${ }^{10}$ Clinical Oncology, Kurmitola General Hospital, Dhaka, Bangladesh; \\ ${ }^{11}$ Department of Radiotherapy, A. Gemelli Hospital, Catholic University, Rome, Italy
}

\begin{abstract}
Aim: To determine the maximum tolerated dose (MTD) of a short-course accelerated radiotherapy and its feasibility for symptomatic palliation of advanced head and neck cancer or head and neck metastases from any primary site. Patients and Methods: A phase I trial in four dose-escalation steps was planned: total dose ranged between 14 and 20 Gy in a total of four fractions administered twice a day. The doselimiting toxicity (DLT) was determined as grade 3 or more toxicity occurring during treatment. The MTD obtained was used to plan a phase II trial. Results: A total of 48 patients were treated. In the phase I trial, the 20 Gy dose level was determined to be the MTD. In the phase II trial, the palliative response rate was $82.7 \%$, with a median duration of palliation of 3 months. Conclusion: Short-course accelerated radiotherapy was well tolerated and effective for palliation. These findings may help design future prospective randomized studies.
\end{abstract}

*These Authors contributed equally to this study.

Correspondence to: Gabriella Macchia, Radiotherapy Unit, Department of Oncology, Università Cattolica del Sacro Cuore, Largo A. Gemelli 1, 86100 Campobasso, Italy. Tel: +39 0874312261, Fax: +390874312720, e-mail: radiationtherapy.ef@gmail.com

Key Words: Head and neck cancer, radiotherapy, palliative care, pain, quality of life.
Locally advanced or metastatic head and neck (H\&N) cancer may present a challenge to the clinician. This disease has an impact on the patient's quality of life (QoL) because of symptoms such as pain, dysphagia, odynophagia, otalgia, bleeding, cough, hoarseness, and respiratory distress (1). In patients with multiple co-morbidities precluding curative treatment, or for those with metastatic disease, palliative radiotherapy (RT) is an effective therapeutic option for reducing symptoms and improving QoL (2-4).

Ideally, palliative RT should be of short duration in order to minimize patient discomfort and treatment cost. A short RT course could also allow for early initiation of chemotherapy for patients with distant metastases and potentially improve survival for those patients. In addition, improvement of patient symptoms with a short RT course may improve patient QoL before their admission or referral to hospice care.

Hypo-fractionation has traditionally been delivered to shorten a treatment course. A higher dose per fraction can be effective in achieving a rapid biological effect with tumor shrinkage and symptom relief (4). However, there is also a higher risk for long-term complications if the tumor is close to a radiosensitive structure. In patients with a short life expectancy because of widespread metastases and poor functional status, long-term complications may not be an issue. Another effective method to shorten treatment time is to deliver RT twice a day. The use of twice daily fractionation reduces the risk of late effects by reducing the fraction size 
(5). This treatment strategy is effective for palliation of pelvic, $\mathrm{H} \& \mathrm{~N}$ tumors, and brain metastases (2, 6-15).

However, accelerated hypo-fractionated RT is not without risk. Severe acute toxicity has been reported and may cause inadvertent deterioration of patient QoL. In order to minimize the prevalence of severe side-effects during treatment, radiation dose escalation may be required in order to establish the dose threshold for severe toxicity.

Thus, the aim of this prospective study was to assess the feasibility of a short course of palliative RT for patients with $\mathrm{H} \& \mathrm{~N}$ cancer using escalating dose increments (dose levels) to determine the maximum tolerated dose (MTD) for severe toxicity. Once the MTD was determined, this dose level was used in a subsequent phase II trial to evaluate the feasibility of accelerated hypo-fractionated RT for palliation of $\mathrm{H} \& \mathrm{~N}$ cancer.

\section{Patients and Methods}

Eligibility criteria. Patients referred to the Radiotherapy Unit of the Catholic University of Sacred Heart in Campobasso (Italy), were required to have histologically proven locally advanced $\mathrm{H} \& \mathrm{~N}$ cancer or $\mathrm{H} \& \mathrm{~N}$ metastases from any primary site. They were excluded from curative therapy because of disease stage, poor performance status, and presence of several comorbidities. Other selection criteria included age $>18$ years, Eastern Cooperative Oncology Group (ECOG) performance status of 3 or less, and no prior RT to the same region. Patients who received systemic therapy were allowed to participate in the trial provided that the last date of chemotherapy was at least 10 days before RT. Pretreatment evaluation included a complete clinical history, physical examination, complete blood count and H\&N computed tomographic (CT) scan. Informations on pain and other symptoms, performance status (ECOG) and QoL were registered at baseline and at each follow-up visit. Pain was measured with Visual Analog self-assessment Scale (VAS) (16). Pain intensity and use of analgesics were also recorded according to the International Atomic Energy Agency (IAEA) scale (Pain and Drug scores) (17). The QoL indices were evaluated using the cancer linear analog scales (CLAS1, CLAS2 and CLAS3) which assess well-being, fatigue and the ability to perform daily activities, respectively. This method is based on a linear analog scale, as reported for pain evaluation. The reliability and validity of these QoL indices were previously demonstrated and reported (18).

Study design. Phase I: A dose-escalation study was designed to define the recommended phase II dose, also referred to as the MTD of an accelerated hypo-fractionated course of RT for H\&N cancer palliation. The recommended phase II dose was defined as the dose level below the highest delivered dose associated with dose-limiting toxicity (DLT) in at least one-third of patients (19). DLT was defined as any acute toxicity of grade 3 or more, according to the Radiation Therapy Oncology Group (RTOG) scale (20). A minimum of six patients were treated at each dose level. If DLT was observed in fewer than two out of six patients at a given dose level (providing that at least 3 months of followup had passed since the sixth patient enrolled at the last dose level completed RT), the trial proceeded to the next dose level. If DLT occurred in two out of six patients at a given dose level, treatment in up to six additional patients was required at this dose level. If DLT occurred in more than two patients of the six-patient cohort, dose escalation was stopped, and the dose level below that was considered the recommended phase II dose. If DLT occurred in four or more patients of the expanded 12-patient cohort, dose escalation was stopped, and the next lower dose level below that was considered the recommended phase II dose. If DLT occurred in fewer than four patients of the expanded 12-patient cohort, the trial proceeded to the next level. As an added precaution for patient safety, we only planned for four dose levels as we were not certain about the long-term effect of RT dose escalation on normal tissue tolerance when the patient might also receive chemotherapy for distant metastases. Among patients who did receive systemic therapy, those who experienced a good response to the combined treatment might have a longer life expectancy and might experience unexpected complications.

Phase II: The sample size was calculated based on the two-stage optimal design by Simon et al. (21). The design tested the null hypothesis that the symptomatic response rate for this population would improve from $10.0 \%$ without RT to $30.0 \%$ with RT, using an $\alpha$ error of 0.05 and a $\beta$ error of 0.2 . Thus, the first step planned to include 10 patients. Enrolment was to be stopped followed by study closure if there was no response. However, in the case of detection of at least one symptomatic response, the study would enrol another 19 patients up to a total number of 29 patients. The regimen would be considered inactive if five or fewer responses out of 29 were recorded.

Treatment. All patients underwent CT scan simulation. Images were taken in 5-mm increments over the region of interest. The gross tumor volume (GTV) was defined as the primary tumor or its metastatic site. The clinical target volume (CTV) was defined as the GTV plus $1 \mathrm{~cm}$ margin. The planning target volume (PTV) was the CTV with a margin of $1 \mathrm{~cm}$ in all directions. Threedimensional conformal RT (3D-CRT) was planned. There were four dose levels of $14 \mathrm{~Gy}$ in $3.5 \mathrm{~Gy}$ fractions twice a day, $16 \mathrm{~Gy}$ in 4 Gy twice a day, 18 Gy in 4.5 Gy twice a day, and 20 Gy in 5 Gy twice a day. Based on a $\alpha / \beta$ ratio of 3 , the equivalent dose in 2 Gy fractions (EQD2) corresponding to these four dose levels was 20.6, 24.1,27.8 and 32.0 Gy, respectively (22). The dose was specified according to the International Commission on Radiation Units and Measurements Report 62 (23). Patients were treated on two consecutive days with twice daily fractionation and an interval of at least 8 hours between fractions to allow normal tissue repair. During treatment planning and delivery, the medical and physics staff performed two independent checks for quality assurance, as previously reported (24). Setup reproducibility was checked before any fraction using electronic portal imaging, as previously described (25).

Follow-up evaluation. Fifteen days after RT, patients underwent the first assessment. Thereafter, patients were followed-up every 2 months with blood count and physical examination. Acute and late toxicities were evaluated with RTOG and the European Organisation for Research and Treatment of Cancer (EORTC)-RTOG toxicity scales, respectively (20). Data concerning symptom relief, pain, drug score and QoL were annotated. Bleeding was considered to be completely palliated if the patient required no further medication to control the symptom. Complete pain relief was defined as a VAS 
Table I. Phase I-II study: patient characteristics (48 patients).

\begin{tabular}{lc}
\hline Characteristic & Value \\
\hline Age (years) & \\
$\quad$ Median & 79.5 \\
$\quad$ Range & $40-98$ \\
Gender, n (\%) & \\
$\quad$ Male & $20(41.7)$ \\
Female & $28(58.3)$ \\
ECOG PS, n (\%) & \\
0 & $10(20.8)$ \\
1 & $13(27.1)$ \\
2 & $15(31.3)$ \\
3 & $10(20.8)$ \\
\hline
\end{tabular}

ECOG PS: Eastern Cooperative Oncology Group performance status.

score of 0 . A reduction, but no complete resolution of symptom severity, or a decrease in pain and drug score were considered to be a partial response. In addition, information about QoL and performance status was compared to those at baseline and graded as improved, stable, or worse.

\section{Results}

Patients' characteristics. This trial was registered as NCT03196700 and all procedures were approved by the local Ethics Committee and were in accordance with the Helsinki Declaration (n. UCSC-CB-2009/31). All patients provided written informed consent before study entry. A total of 48 patients were enrolled in this trial. Patients presented disease stage III-IV not amenable to curative therapies due to poor performance status and multiple comorbidities. Twenty-five patients were enrolled in the phase I study. The six patients enrolled at the fourth dose level were also counted as patients enrolled in the phase II study. Patient characteristics are summarized in Table I. Fifteen and 33 patients had locally advanced and metastatic H\&N cancer, respectively. Primary sites and histopathology are detailed in Table II, and the most frequent baseline symptoms in Table III.

Phase I study. In order to assess acute toxicity properly, patient accrual at every level continued until the sixth enrolled patient had a minimum follow-up of 3 months after RT. Acute toxicities for every cohort of the phase I study are detailed in Table IV. Only 1 patient presented grade 3 mucosal toxicity (severe mucositis) at the fourth dose level. Consequently, the dose level of $20 \mathrm{~Gy}$ was determined to be the MTD. No other patients experienced greater than grade 2 acute toxicity. The treatment was also well tolerated when administered between systemic therapy courses (14 patients, $56.0 \%$ ). No patient died within 30 days of RT completion.
Table II. Phase I-II study: site of primary tumor and histopathology (48 patients).

\begin{tabular}{lcrr}
\hline Site & Histopathology & $\mathrm{n}$ & $\%$ \\
\hline Head and neck & Squamous cell carcinoma & 15 & 31.2 \\
Skin & & 13 & 27.1 \\
& Spinocellular carcinoma & 8 & 16.7 \\
& Melanoma & 4 & 8.3 \\
Ovary & Basocellular carcinoma & 1 & 2.1 \\
& & 4 & 8.3 \\
& Squamous cell carcinoma & 2 & 4.1 \\
Breast & Transitional cell carcinoma & 2 & 4.1 \\
& & 3 & 6.2 \\
& & 1 & 2.1 \\
Lung & Ductal carcinoma & 2 & 4.1 \\
& Carcinoma (not defined) & 3 & 6.2 \\
Thyroid & & 1 & 2.1 \\
Prostate & Adenocarcinoma & 2 & 4.1 \\
Uterus & Squamocellular carcinoma & 1 & 2.1 \\
Bladder & Papillary carcinoma & 1 & 2.1 \\
Kidney & Adenocarcinoma & 1 & 2.1 \\
Bowel & Poorly differentiated carcinoma & 1 & 2.1 \\
Rectum & Urothelial cell carcinoma & 1 & 2.1 \\
Pancreas & Clear cell carcinoma & 1 & 2.1 \\
Soft tissue & Adenocarcinoma & 1 & 2.1 \\
Squamous cell & Adenocarcinoma & 1 & 2.1 \\
of unknown primary & Adenocarcinoma & 1 & 2.1 \\
\hline & Angiosarcoma & & \\
& & 1 & 2.1 \\
\hline
\end{tabular}

Table III. Phase I-II study: main baseline symptoms (48 patients).

\begin{tabular}{lrr}
\hline Symptom & $\mathrm{n}$ & $\%$ \\
\hline Pain & 32 & 66.6 \\
Odynophagia & 8 & 16.7 \\
Bleeding-pain & 4 & 8.3 \\
Bleeding alone & 2 & 4.2 \\
Dysphagia & 2 & 4.2 \\
\hline
\end{tabular}

Phase II study. In the first stage of the trial's design, 10 patients were enrolled and treated with $20 \mathrm{~Gy}$. Four patients (40.0\%) experienced complete symptom remission, five patients $(50.0 \%)$ had partial symptom remission, and there was no change in one patient $(10.0 \%)$. Thus, the palliative response rate (complete plus partial symptom remission) in the first stage was $90.0 \%$. In the second stage, another 19 patients were enrolled to reach the total required number of 29. The overall palliative response rate (complete plus partial symptom remission) for the whole group was $82.7 \%$ (95\% CI $=65.0 \%-92.9 \%)$. Median duration of palliation was 3 months. Data regarding symptomatic response are 
Table IV. Phase I study: acute toxicity.

\begin{tabular}{|c|c|c|c|c|c|c|c|c|c|}
\hline \multirow[t]{2}{*}{ Dose level } & & \multicolumn{2}{|c|}{$\begin{array}{c}1 \\
(14 \mathrm{~Gy})\end{array}$} & \multicolumn{2}{|c|}{$\begin{array}{c}2 \\
(16 \mathrm{~Gy})\end{array}$} & \multicolumn{2}{|c|}{$\begin{array}{c}3 \\
(18 \mathrm{~Gy})\end{array}$} & \multicolumn{2}{|c|}{$\begin{array}{c}4 \\
(20 \mathrm{~Gy})\end{array}$} \\
\hline & & No. & $\%$ & No. & $\%$ & No. & $\%$ & No. & $\%$ \\
\hline Enrolled patients & & 7 & 100 & 6 & 100 & 6 & 100 & 6 & 100 \\
\hline Acute toxicity & Grade & & & & & & & & \\
\hline \multirow{2}{*}{ Skin } & 1 & 1 & 14.3 & 2 & 33.3 & 1 & 16.7 & 2 & 33.3 \\
\hline & 2 & 2 & 28.6 & 1 & 16.7 & 0 & 0.0 & 0 & 0.0 \\
\hline \multirow[t]{3}{*}{ Mucosae } & 1 & 2 & 28.6 & 1 & 16.7 & 0 & 0.0 & 1 & 16.7 \\
\hline & 2 & 0 & 0.0 & 1 & 16.7 & 1 & 16.7 & 1 & 16.7 \\
\hline & 3 & 0 & 0.0 & 0 & 0.0 & 0 & 0.0 & 1 & 16.7 \\
\hline \multirow[t]{2}{*}{ Pharynx } & 1 & 2 & 28.6 & 0 & 0.0 & 0 & 0.0 & 1 & 16.7 \\
\hline & 2 & 0 & 0.0 & 0 & 0.0 & 3 & 50.0 & 2 & 33.3 \\
\hline Eye & 2 & 1 & 14.3 & 0 & 0.0 & 0 & 0.0 & 0 & 0.0 \\
\hline Patients experiencing DLT & & 0 & 0.0 & 0 & 0.0 & 0 & 0.0 & 1 & 16.7 \\
\hline
\end{tabular}

DLT: Dose-limiting toxicity.

reported in Table V. The response rate for pain was $81.5 \%$ (95\% CI=62.8\%-92.3\%). The mean pre- and post-treatment VAS was 4.7 and 2.2 , respectively $(p<0.001)$. For pain relief alone or associated with other symptoms, nine out of 27 patients $(33.3 \%)$ had complete symptom resolution (VAS=0), 13 out of $27(48.1 \%)$ had partial symptom resolution $(\triangle \mathrm{VAS}$ : median $=50.0 \%$, range $=16.7 \%-66.0 \%)$, four out of $27(14.8 \%)$ had no change, and one out of 27 $(3.7 \%)$ experienced increased pain $(\Delta \mathrm{VAS}=33.3 \%)$. Fifty percent of patients with dysphagia (alone or associated with pain) reported complete symptom resolution, $25.0 \%$ had a partial resolution, and $25.0 \%$ experienced no change. Complete and partial bleeding resolution was observed in $16.7 \%$ and $83.3 \%$ of the patients respectively. At the first follow-up, $44.8 \%$ of patients presented an improved ECOG performance status, $41.4 \%$ were stable, and $13.8 \%$ experienced deterioration. Overall, most patients $(93.1 \%)$ were able to classify the different aspects of QoL. An improvement of CLAS1, CLAS2 and CLAS3 indices was noted in $22.2 \%, 18.5 \%$, and $22.2 \%$ of patients, while a stability of their values was recorded in $40.7 \%, 33.3 \%$, and $29.6 \%$, respectively.

Late toxicity. At a median follow-up time of 4 months (range $=1-16$ months), only one patient $(2.1 \%$ ) experienced grade 3 late skin toxicity (marked atrophy and telangiectasia) at $20 \mathrm{~Gy}$. Another patient $(2.1 \%)$ developed late grade 2 skin toxicity (patch atrophy and moderate telangiectasia) at 20 Gy. Only five patients $(10.4 \%)$ had grade 1 late skin toxicity (slight atrophy and pigmentation change) at $18 \mathrm{~Gy}(\mathrm{n}=1)$, and 20 Gy $(n=4)$ respectively.
Table V. Phase II study: symptomatic response.

\begin{tabular}{lrr}
\hline Response & No. & $\%$ \\
\hline Partial symptom remission & 16 & 55.2 \\
Complete symptom remission & 8 & 27.6 \\
No change & 4 & 13.8 \\
Progression of symptoms & 1 & 3.4 \\
\hline
\end{tabular}

\section{Discussion}

To our knowledge, this is the first radiation dose-escalation study to determine the MTD for effective palliation of $H \& N$ cancer. The results of our study have significant implications as this approach may also reduce treatment cost, which is also a limiting factor in developed countries $(26,27)$.

We demonstrated that an accelerated hypo-fractionated RT of 20 Gy effectively reduced pain, bleeding and dysphagia, and improved QoL in patients with $H \& N$ cancer with a short life expectancy because of metastatic disease or poor performance status. Our response rate of $82.7 \%$ was among the best in the literature. Our regimen was also very well tolerated, with minimum grade 3 toxicity. We did not increase the dose beyond 20 Gy because of the concern regarding long-term toxicity as a significant percentage of the patients also received chemotherapy. Those who responded to chemotherapy may be long-term survivors and develop long-term complications. 
The data in our study compared favorably with other RT regimens frequently used for palliation of $\mathrm{H} \& \mathrm{~N}$ cancer. Mohanti et al. reported up to $59.0 \%$ palliation of symptoms in 505 patients with stage IV H\&N cancer treated with a hypo-fractionated course of $20 \mathrm{~Gy}$ in five fractions over 1 week (28). A similar regimen to a higher dose (median 30 Gy) provided up to $79.0 \%$ response rate in patients with H\&N cancer undergoing palliative RT (29). However, despite effective palliation, such a prolonged treatment course is time consuming and inconvenient for the patients if they were to receive hospice care. Thus, a shorter course of RT may be more cost effective and may reduce patient need for transportation if it provides equal palliation.

An accelerated course of twice a day over two consecutive days (' $2 \times 2$ ', also known as Quad Shot) has been reported to achieve a good response in patients with $\mathrm{H} \& \mathrm{~N}$ cancer undergoing RT for symptom palliation. The Quad Shot technique was proposed initially by the MD Anderson Cancer Center and was adopted later by the RTOG for palliation of gynecological malignancies. The protocol was then applied for palliation of H\&N cancer (2, 8-14). In those studies, the doses per cycle ranged from 14 to $14.8 \mathrm{~Gy}$, delivered in four fractions (twice a day for 2 consecutive days). Several techniques of RT ranging from intensitymodulated radiation therapy (IMRT), 3D-CRT, and ${ }^{60} \mathrm{Co}$ teletherapy were employed for palliation (2,8-14). The Quad Shot regimen achieved a good response and adequate survival rates with acceptable acute toxicity. However, although those studies tested the $2 \times 2$ regimen in this setting, our study was the first to systematically assess the MTD.

Lok et al. reported a $65.0 \%$ response rate in patients with $75 \mathrm{H} \& \mathrm{~N}$ cancer treated by IMRT with the Quad Shot regimen to a total dose of $44.4 \mathrm{~Gy}$ in three cycles of $3.7 \mathrm{~Gy}$ twice a day on two consecutive days. Only $37.0 \%$ of the patients completed three cycles. The treatment was well tolerated with $5.0 \%$ grade 3 toxicity (13). Given the fact that IMRT may not be available in emerging countries, our treatment protocol represents a safe and effective method of palliation for patients with $\mathrm{H} \& \mathrm{~N}$ cancer. Moreover, our fractionation also allows the early initiation of systemic therapy, which remains the main treatment for patients with distant metastases. For example, $56.0 \%$ of the patients in our study received systemic therapy with no major advents related to therapies. In patients who required chemotherapy as part of their treatment protocol, early initiation of chemotherapy is associated with better survival compared to delayed chemotherapy (30). Thus, our treatment protocol may serve as a template for future prospective studies combining chemotherapy and RT for patients with metastatic $\mathrm{H} \& \mathrm{~N}$ cancer.

In conclusion, the results of this trial demonstrated that our protocol is safe, effective in terms of response, and may possibly improve survival due to allowing early initiation of chemotherapy in patients with distant metastases of $\mathrm{H} \& \mathrm{~N}$ cancer. Based on the results of this study, a multicenter phase III trial has been planned to compare this scheme with a traditional palliative regimen of $30 \mathrm{~Gy}$ in 10 fractions.

\section{Conflicts of Interest}

None.

\section{Funding}

This research did not receive any specific grant from funding agencies in the public, commercial, or not-for-profit sector.

\section{Acknowledgements}

The Authors would like to thank Dayleen De Riggs for her help in editing the article.

\section{References}

1 Agarwal JP, Nemade B, Murthy V, Ghosh-Laskar S, Budrukkar A, Gupta T, D'Cruz A, Pai P, Chaturvedi P and Dinshaw K: Hypofractionated, palliative radiotherapy for advanced head and neck cancer. Radiother Oncol 89: 51-56, 2008.

2 Finnegan TS, Bhatt NH, Shaughnessy JN, Perez C, Redman R, Silverman C, Bumpous J, Potts K and Dunlap NE: Cyclical hypofractionated radiotherapy technique for palliative treatment of locally advanced head and neck cancer: institutional experience and review of palliative regimens. J Community Support Oncol 14: 29-36, 2016.

3 Fortin B, Khaouam N, Filion E, Bguyen-Tan PF, Bujold A and Lambert L: Palliative radiation therapy for advanced head and neck carcinomas: a phase 2 study. Int J Radiat Oncol Biol Phys 95: 647-653, 2016.

4 Bledsoe TJ, Noble AR, Reddy CA, Burkey BB, Greskovich JF, Nwizu T, Adelstein DJ, Saxton JP and Koyfman SA: Splitcourse accelerated hypofractionated radiotherapy (SCAHRT): a safe and effective option for head and neck cancer in the elderly or infirm. Anticancer Res 36: 933-940, 2016.

5 Rades D, Seidl D, Wollenberg B, Schild SE and Hakim SG: Radiochemotherapy with paclitaxel for recurrent previously irradiated squamous cell carcinoma of the head and neck. Anticancer Res 36: 5463-5468, 2016.

6 Spanos W Jr., Guse C, Perez C, Grigsby P, Doggett RL and Poulter C: Phase II study of multiple daily fractionations in the palliation of advanced pelvic malignancies: preliminary report of RTOG 8502. Int J Radiat Oncol Biol Phys 17: 659-661, 1989.

7 Caravatta L, Padula GDA, Macchia G, Ferrandina G, Bonomo P, Deodato F, Massaccesi M, Mignogna S, Tambaro R, Rossi M, Flocco M, Scapati A, Scambia G, Pacelli F, Valentini V, Cellini $\mathrm{N}$ and Morganti AG: Short course accelerated radiation therapy (SHARON) in palliative treatment of advanced pelvic malignancies: a phase I study. Int J Radiat Oncol Biol Phys 83: e627-631, 2012.

8 Paris KJ, Spanos WJ Jr., Lindberg RD, Jose B and Albrink F: Phase I-II study of multiple daily fractions for palliation of advanced head and neck malignancies. Int J Radiat Oncol Biol Phys 25: 657-660, 1993. 
9 Corry J, Peters LJ, Costa ID, Milner AD, Fawns H, Rischin D and Porceddu S: The 'QUAD SHOT' - a phase II study of palliative radiotherapy for incurable head and neck cancer. Radiother Oncol 77: 137-142, 2005.

10 Carrascosa LA, Yashar CM, Paris KJ, Larocca RV, Faught SR and Spanos WJ: Palliation of pelvic and head and neck cancer with paclitaxel and a novel radiotherapy regimen. J Palliat Med 10: 877-881, 2007.

11 Chen AM, Vaughan A, Narayan S and Vijayakumar S: Palliative radiation therapy for head and neck cancer: toward an optimal fractionation scheme. Head Neck 30: 1586-1591, 2008.

12 Ghoshal S, Chakraborty S, Moudgil N, Kaur M and Patel FD: Quad Shot: a short but effective schedule for palliative radiation for head and neck carcinoma. Indian J Palliat Care 15: 137-140, 2009.

13 Lok BH, Jiang G, Gutiontov S,Lanning RM, Sridhara S, Sherman EJ, Tsai CJ, McBride SM, Riaz N and Lee NY: Palliative head and neck radiotherapy with the RTOG 8502 regimen for incurable primary or metastatic cancers. Oral Oncol 51: 957-962, 2015

14 Gamez ME, Agarwal M, Hu KS, Lukens JN and Harrison LB: Hypofractionated palliative radiotherapy with concurrent radiosensitizing chemotherapy for advanced head and neck cancer using the 'Quad-Shot regimen'. Anticancer Res 37: 685-691, 2017.

15 Caravatta L, Deodato F, Ferro M, Macchia G, Massaccesi M, Cilla S, Tambaro R, Mignogna S, Padula GD, Musacchio M, Flocco M, Cantore G, Scapati A, Bogale S, Balducci M, Valentini V, Cellini N and Morganti AG: Results of a phase II study of short-course accelerated radiation therapy (SHARON) for multiple brain metastases. Am J Clin Oncol 38: 395-400, 2015.

16 Melzack R: The McGill Pain Questionnaire: major properties and scoring methods. Pain 1: 277-299, 1975.

17 Salazar OM, Sandhu T, da Motta NW, Escutia MA, LanzósGonzales E, Mouelle-Sone A, Moscol A, Zaharia M and Zaman S: Fractionated half-body irradiation (HBI) for the rapid palliation of widespread, symptomatic, metastatic bone disease: a randomized phase III trial of the International Atomic Energy Agency (IAEA). Int J Radiat Oncol Biol Phys 50: 765-775, 2001.

18 Sutherland HJ, Walker P and Til JE: The development of a method for determining oncology patients' emotional distress using linear analogue scales. Cancer Nurs 11: 303-308, 1988.

19 Arbuck SG: Workshop on phase I study design. Ninth NCI/EORTC New Drug Development Symposium, Amsterdam. Ann Oncol 7: 567-573, 1996.

20 Cox JD, Stetz J and Pajak TF: Toxicity criteria of the Radiation Therapy Oncology Group (RTOG) and the European Organization for Research and Treatment of Cancer (EORTC). Int J Radiat Oncol Biol Phys 31: 1341-1346, 1995.
21 Simon R, Wittes RE and Ellenberg SS: Randomized phase II clinical trials. Cancer Treatment Rep 69: 1375-1381, 1985.

22 Withers HR: Biological basis of radiation therapy. In: Principles and Practice of Radiation Oncology. Perez CA and Brady LW (eds.). Philadelphia, Lippincott, pp. 64-69, 1992.

23 International Commission on Radiation Units and Measurements, ICRU report 62: Prescribing, Recording and Reporting Photon Beam Therapy (supplement to ICRU report 50). Bethesda, 1999

24 Morganti AG, Deodato F, Zizzari S, Cilla S, Digesu' C, Macchia G, Panunzi S, De Gaetano A, Piermattei A, Cellini N and Valentini V: Complexity index (COMIX) and not type of treatment predicts undetected errors in radiotherapy planning and delivery. Radiother Oncol 89: 320-329, 2008

25 Deodato F, Cilla S, Massaccesi M, Macchia G, Ippolito E, Caravatta L, Picardi V, Romanella M, Di Falco C, Bartollino A, Valentini V, Cellini N, De Spirito M, Piermattei A and Morganti AG: Daily on-line setup correction in 3D-conformal radiotherapy: Is it feasible? Tumori 98: 441-444, 2012.

26 Jeremic B, Vanderpuye V, Abdel-Wahab S, Gaye P, Kochbati L, Diwani M, Emwula P,Oro B, Lishimpi K, Kigula-Mugambe J, Dawotola D, Wondemagegnehu T, Nyongesa C, Oumar N, ElOmrani A, Shuman T, Langenhoven L and Fourie L: Patterns of practice in palliative radiotherapy in Africa - case revisited. Clin Oncol 26: 333-343, 2014.

27 Egestad $\mathrm{H}$ and Nieder C: Undesirable financial effects of head and neck cancer radiotherapy during the initial treatment period. Int J Circumpolar Health 74: 26686, 2015.

28 Mohanti BK, Umapathy H, Bahadur S, Thakar A and Pathy S: Short course palliative radiotherapy of $20 \mathrm{~Gy}$ in 5 fractions for advanced and incurable head and neck cancer: AIIMS study. Radiother Oncol 71: 275-280, 2004.

29 Teckie S, Lok BH, Rao S, Gutiontov SI, Yamada Y, Berry SL, Zelefsky MJ and Lee NY: High dose hypo-fractionated radiotherapy is effective and safe for tumors in the head and neck. Oral Oncol 60: 74-80, 2016.

$30 \mathrm{Kim}$ HW, Lee JC, Lee J, Kim JW, Kim J and Hwang JH: Early versus delayed initiation of adjuvant treatment for pancreatic cancer. PLoS One 12: e0173960, 2017. 